\title{
A New Drug-Drug Interaction Between Hydroxychloroquine and Metformin? A Signal Detection Study
}

\author{
Jean-Louis Montastruc ${ }^{1}$ (D) Pierre-Louis Toutain ${ }^{2,3}$
}

Published online: 3 June 2020

(c) Springer Nature Switzerland AG 2020

\begin{abstract}
Introduction Hydroxychloroquine was recently promoted in patients infected with COVID-19 infection. A recent experimental study has suggested an increased toxicity of hydroxychloroquine in association with metformin in mice.

Objective The present study was undertaken to investigate the reality of this putative drug-drug interaction between hydroxychloroquine and metformin using pharmacovigilance data.

Methods Using VigiBase ${ }^{\circledR}$, the WHO pharmacovigilance database, we performed a disproportionality analysis (case/noncase study). Cases were reports of fatal outcomes with the drugs of interest and non-cases were all other reports for these drugs registered between 1 January 2000 and 31 December 2019. Data with hydroxychloroquine (or metformin) alone were compared with the association hydroxychloroquine + metformin. Results are reported as ROR (reporting odds ratio) with their $95 \%$ confidence interval.

Results Of the 10,771 Individual Case Safety Reports (ICSR) involving hydroxychloroquine, 52 were recorded as 'fatal outcomes'. In comparison with hydroxychloroquine alone, hydroxychloroquine + metformin was associated with an ROR value of 57.7 (23.9-139.3). In comparison with metformin alone, hydroxychloroquine + metformin was associated with an ROR value of 6.0 (2.6-13.8).

Conclusion Our study identified a signal for the association hydroxychloroquine + metformin that appears to be more at risk of fatal outcomes (particularly by completed suicides) than one of the two drugs when given alone.
\end{abstract}

\section{Introduction}

Hydroxychloroquine is an antimalarial drug, today widely used in rheumatoid arthritis or systemic lupus erythematous [1], that was recently proposed to treat COVID-19 infections $[2,3]$, despite lack of clinical evidence [4]. Besides efficacy, its clinical pharmacology (safety, drug-drug interactions) particularly in COVID-19 patients is not well known. In fact, a recent non-peer-reviewed publication (available in a

Jean-Louis Montastruc

jean-louis.montastruc@univ-tlse3.fr

1 Service de Pharmacologie Médicale et Clinique, Centre de PharmacoVigilance, de Pharmacoépidémiologie et d'Informations sur le Médicament, Faculté de Médecine, Centre Hospitalier Universitaire, 37 allées Jules-Guesde, 31000 Toulouse, France

2 INTHERES, Université de Toulouse, INRA, ENVT, Toulouse, France

3 The Royal Veterinary College, University of London, London, UK preprint server) from American oncologists described fatal toxicity of hydroxychloroquine (or of chloroquine) in association with metformin in mice [5].

\section{Objective}

These aforementioned results led us to investigate the reality of this putative drug-drug interaction in humans using a pharmacovigilance database. In fact, previous studies have shown that pharmacovigilance databases can be successfully used to detect drug-drug interactions [6].

\section{Methods}

The study was performed in VigiBase ${ }^{\circledR}$, the WHO pharmacovigilance database, which registers all Individual Case Safety Reports (ICSRs) from more than 130 countries around the world. ICSRs are entered into VigiBase ${ }^{\circledR}$ after rigorous quality checks and deduplication [7]. 


\section{Key Points}

Following a recent non-peer-reviewed publication describing fatal toxicity of hydroxychloroquine in association with metformin in mice, the present study was undertaken to investigate this putative drug-drug interaction between hydroxychloroquine and metformin using pharmacovigilance data.

Using VigiBase ${ }^{\circledR}$, the WHO pharmacovigilance database, we found an increased risk of fatal outcomes with the association in comparison with each of the two drugs when used alone.

This study suggests a pharmacovigilance signal of fatal outcome with the association hydroxychloroquine + metformin that should be confirmed by further studies.

We extracted all ICSRs registered between 1 January 2000 and 31 December 2019 as 'fatal outcomes' [High Level Group Term (HLGT) in the System Organ Class (SOC) group 'General Disorders and Administration Site Conditions' according to the Medical Dictionary for Regulatory Activities $\left(\right.$ MedDRA $\left.^{\circledR}\right)$ ] in patients aged between 18 and 64 years. We excluded ICSRs from patients with sex unknown as well as those $<18$ years and $>64$ years because in a preliminary analysis of VigiBase ${ }^{\circledR}$ we failed to find any fatal outcome with the drug association (hydroxychloroquine + metformin) in patients aged $<18$ and $>64$ years. Following current protocols to detect a pharmacovigilance signal in large pharmacovigilance databases [8], we performed a disproportionality analysis (case/non-case analysis) [8], with cases being occurrence of 'fatal outcomes' with hydroxychloroquine (or chloroquine) (P01BA according to ATC classification of drugs) and non-cases all other ICSRs with hydroxychloroquine (or chloroquine) alone. Data with hydroxychloroquine (or chloroquine) alone were compared with the association hydroxychloroquine (or chloroquine) + metformin (A10BA). We also performed such analyses including other drugs used in diabetes mellitus; that is, sulfonylureas (A10BB), alpha glucosidase inhibitors (A10BF), thiazolidinediones (A10BG), dipeptidyl peptidase 4 (DPP-4) inhibitors (A10BH), glucagon like peptide-1 (GLP-1) analogues (A10BJ) and sodium glucose cotransporter 2 (SGLT2) inhibitors (A10BK).

Results are presented as reporting odds ratio (ROR), a concept similar to OR in case control studies, with its $95 \%$ confidence interval $(\mathrm{CI})$.

\section{Results}

Of the 21,580,375 deduplicated ICSRs recorded in VigiBase $^{\circledR}, 7,961,942$ met the inclusion criteria defined above with 10,771 involving hydroxychloroquine and 52 recorded as 'fatal outcomes' with this drug. These 52 ICSRs were registered mainly in women $(78.8 \%)$, with the main age group 45-64 years (55.8\%). The majority came from the United States of America (US) (80.8\%). Among these 52 ICSRs, 30 were described as 'completed suicides'. With metformin, 1413 deaths were found (including 1083 suicides), $58.6 \%$ in men, mainly coming from the US (94.1\%). The most frequent age group was 45-64 years (65.6\%). Seven deaths were registered with the association hydroxychloroquine + metformin with three reports in individuals aged 18-44 years and four aged 45-64 years. Four were observed in men and six were registered as completed suicides. All were from the US (Table 1).

For the total fatal reports, an ROR value of 57.7 (23.9-139.3) was associated with hydroxychloroquine + metformin in comparison with hydroxychloroquine alone. In comparison with metformin alone, the association hydroxychloroquine + metformin was associated with ROR 6.0 (2.6-13.8). After excluding suicides, similar results were obtained for the association hydroxychloroquine + metformin versus hydroxychloroquine alone: ROR 15.8 (2.1-120.6).

No ICSR combining hydroxychloroquine and other drugs used in diabetes (sulfonylureas, alpha glucosidase inhibitors, thiazolidinediones, DPP-4 inhibitors, GLP-1 analogues, SGLT2 inhibitors) was found in VigiBase ${ }^{\circledR}$.

Table 1 Number of total, fatal, non-suicidal reports and their ratios registered with hydroxychloroquine, metformin and their association in VigiBase $^{\circledR}$, the WHO pharmacovigilance database, between 1 January 2000 and 31 December 2019

\begin{tabular}{rrrll} 
Total reports & Fatal reports & $\begin{array}{l}\text { Non-suicidal fatal } \\
\text { reports }\end{array}$ & $\begin{array}{l}\text { Suicidal fatal/non-suicidal } \\
\text { fatal ratio }((\%)\end{array}$ & $\begin{array}{l}\text { Non-suicidal fatal/ } \\
\text { total reports ratio } \\
(\%)\end{array}$ \\
\hline 10,771 & 52 & 22 & 136 & 0.20 \\
31,582 & 1413 & 330 & 328 & 0.10 \\
32 & 7 & 1 & 600 & 3.12
\end{tabular}


For chloroquine, 15 deaths were registered $(60.0 \%$ in women, $73.3 \%$ aged $18-44$ years, including eight completed suicides. No fatal outcome was registered for the association chloroquine + metformin (or other drugs used in diabetes).

\section{Discussion}

The present study was performed to search for a possible drug-drug interaction between hydroxychloroquine + metformin related to the recently developed off-label use of hydroxychloroquine in the field of COVID-19. In addition, some studies have investigated hydroxychloroquine in association with metformin in diabetic patients to achieve a better control of type- 2 diabetes conditions [10]. Conversely, metformin was studied as an adjunctive therapy for systemic lupus erythematous, a condition for which hydroxychloroquine is routinely administered [11]. In fact, our study was prompted by the paper from Rajeshkumar's group [5] showing a higher mortality ratio (30-40\%) in mice treated with hydroxychloroquine + metformin in comparison with the control group receiving metformin alone. The authors found an increased number of autophagosomes in the heart, liver and kidneys of mice treated with the combination. They did not conclude about a univocal mechanism for their observation but suggested a synergistic effect between metformin, an inhibitor of the mitochondrial complex I, and hydroxychloroquine, which inhibits autophagy [5].

Our study did not allow for discussion of the possible mechanism of action but shows a safety signal with the association hydroxychloroquine + metformin. We found that more reports of fatal outcomes (and especially suicides) are registered in VigiBase ${ }^{\circledR}$ with the association than with each of the drugs administered individually. No conclusion can be made for chloroquine due to the absence of lethal reports with the association.

Of course, our work suffers from the classical biases of all pharmacovigilance studies: under- or selective reporting and lack of systematic information on doses and duration of exposure in VigiBase ${ }^{\circledR}$. Of course, it is important to underline that ROR investigates the risk of ADR reporting rather than a true risk. Our study was not performed in COVID19 patients but in those receiving hydroxychloroquine for rheumatic or dermatologic diseases. However, there is no reason to believe that the described drug-drug interaction could depend on the underlying disease(s). It was not possible to perform direct adjustments due to the characteristics of VigiBase ${ }^{\circledR}$. However, Seabroke's group found that in large pharmacovigilance databases, subgroup analyses (as performed in this study) improve both sensitivity and precision and are clearly beneficial over crude analyses $[12,13]$.

The fact that the completed suicide/non-suicidal ratio is higher for the association than with the drugs alone could suggest an additional toxicity of hydroxychloroquine and metformin taken together. Since hydroxychloroquine doses are higher for COVID-19 than for lupus or rheumatoid arthritis, this might increase the significance of the signal.

In contrast, the results of the present study have several important strengths. They are reflective of clinical conditions and cases were retrieved from the world's largest pharmacovigilance database. It used a validated method for the first detection of pharmacovigilance signals [7-9]. We found high significant values of ROR suggesting a true association between the investigated factors. Our results extend the conclusions obtained in animals by Rajeshkumar et al. [5] and should be considered as a meaningful pharmacovigilance signal of a drug-drug interaction between hydroxychloroquine and metformin. In fact, metformin is a widely used drug, particularly in aged and/or obese patients that are found to be particularly at risk for COVID-19 pandemic infection [14].

\section{Conclusion}

The present study found a pharmacovigilance signal that should be confirmed by other studies using other methods for drug-drug interaction detection. Our data suggest that the association increases the risk of death, and particularly death by completed suicide. From a clinical point of view, our results suggest a warning for metformin-treated diabetic patients receiving hydroxychloroquine, for example as selfmedication or in off-label use for COVID-19.

Acknowledgements The authors acknowledge the WHO Uppsala Monitoring Center, which allowed the authors access to VigiBase ${ }^{\circledR}$. The conclusions of the authors are not necessarily those of the WHO Uppsala Monitoring Center or the French Drug Agency (Agence Nationale De Sécurité du Médicament et des produits de santé, ANSM).

Author Contributions JLM and PLT designed the study. JLM extracted the data from the database and performed the statistical analysis. All the authors analysed the data. JLM wrote the paper. The two authors reviewed the successive versions of the manuscript and approved the final version.

\section{Compliance with Ethical Standards}

Conflict of interest The authors declare that they have no competing interests.

Ethical standards According to the clinical research French law, review from an ethics committee is not required for such observational studies. As all data from VigiBase were deidentified, patient informed consent was not necessary.

Funding None. The work was performed during the authors' university hours using VigiBase ${ }^{\circledR}$, which is freely available in the authors' department. 


\section{References}

1. Vinetz JM. Chemotherapy of malaria. In: Brunton LL, Hilal-Dandan R, Knollmann BJ, editors. Goodman and Gilman's the pharmacological basis of therapeutics. 13th ed. New York: McGraw Hill; 2018. p. 968-986.

2. Moore N. Chloroquine for COVID-19 infection. Drug Saf. 2020. https://doi.org/10.1007/s40264-020-00933-4.

3. Roustit M, Guilhaumou R, Molimard M, Milou Drici M, Laporte S, Montastruc JL, On behalf of the French Society of Pharmacology, and Therapeutics (SFPT). Chloroquine and hydroxychloroquine in the management of COVID-19: much kerfuffle but little evidence. Therapie. 2020. https://doi.org/10.1016/j.thera p.2020.05.010Reference.

4. Lenzer J. Covid-19: US gives emergency approval to hydroxychloroquine despite lack of evidence. BMJ. 2020;369:m1335.

5. Rajeshkumar NV, Yabuuchi S, Pai SG, Maitra A, Hidalgo M, Dang CV. Fatal toxicity of chloroquine or hydroxychloroquine with metformin in mice. Biorxiv. 2020. https://doi. org/10.1101/2020.03.31.018556.

6. Van Puijenbroek EP, Egberts AC, Heerdink ER, Leufkens HG. Detecting drug-drug interactions using a database for spontaneous adverse drug reactions: an example with diuretics and non-steroidal anti-inflammatory drugs. Eur J Clin Pharmacol. 2000;56:733-8.

7. Bihan K, Lebrun-Vignes B, Funck-Brentano C, Salem JE. Uses of pharmacovigilance databases: an overview. Therapie. 2020. https ://doi.org/10.1016/j.therap.2020.02.022.
8. Moore N, Berdaï D, Blin P, Droz C. Pharmacovigilance-the next chapter. Therapie. 2019;74:557-67.

9. Faillie JL. Case-non-case studies: principle, methods, bias and interpretation. Therapie. 2019;74:225-32.

10. Purkait I, Pareek A, Arunachalam Panneerselvam SR, Mukhopadhyay MK, Kumar SR, Chandratreya SA. Effectiveness of hydroxychloroquine (HCQ) $400 \mathrm{mg}$ in uncontrolled T2D patients on dual therapy of metformin and sulfonylurea: a real-world experience in India. Diabetes. 2019. https://doi.org/10.2337/db19-1189-P.

11. Tan MKX, Heng TYJ, Mak A. The potential use of metformin, dipyridamole, $N$-acetylcysteine and statins as adjunctive therapy for systemic lupus erythematosus. Cells. 2019;8(4):E323. https:// doi.org/10.3390/cells8040323.

12. Candore G, Juhlin K, Manlik K, et al. Comparison of statistical signal detection methods within and across spontaneous reporting databases. Drug Saf. 2015;38:577-87.

13. Seabroke S, Candore G, Juhlin K, et al. Performance of stratified and subgrouped disproportionality analyses in spontaneous databases. Drug Saf. 2016;39:355-64.

14. Grasselli G, Zangrillo A, Zanella A, Antonelli M, Cabrini L, Castelli A, Cereda D, Coluccello A, Foti G, COVID-19 Lombardy ICU Network. Baseline characteristics and outcomes of 1591 patients infected with SARS-CoV-2 admitted to ICUs of the Lombardy Region, Italy. JAMA. 2020. https://doi.org/10.1001/ jama.2020.5394. 